RESEARCH ARTICLE

\title{
The Relationship between Retinal Ganglion Cell Damage with Du- ration of Diabetes and Diabetic Retinopathy Status
}

\author{
Burcu Nurözler Tabakcl ${ }^{*}$, Gülizar Demirok², Yasemin Topalak ${ }^{3}$ and Ahmet Şengün ${ }^{3}$ \\ ${ }^{1}$ Department of Ophthalmology, Faculty of Medicine, Süleyman Demirel University, Turkey \\ ${ }^{2}$ Department of Ophthalmology, Ankara Training and Research Hospital, Turkey \\ ${ }^{3}$ Department of Ophthalmology, Faculty of Medicine, Ufuk University, Turkey
}

*Corresponding author: Burcu Nurözler Tabakcı, Department of Ophthalmology, Faculty of Medicine, Süleyman Demirel University, Ankara, Turkey, E-mail: burcunurtabakci@gmail.com

\begin{abstract}
Purpose: We determined whether Diabetes Mellitus (DM) affects the retinal Ganglion Cell-Inner Plexiform Layer (GCl$\mathrm{PL}$ ) thickness in patients who have no Diabetic Retinopathy (DR) or Non-Proliferative Diabetic Retinopathy (NPDR).

Design: Retrospective, comparative study.

Methods: Of $60 \mathrm{DM}$ patients, 56 eyes with no DR (group 1) and 52 eyes with NPDR (group 2), and 104 eyes of 60 healthy subjects underwent the Ganglion Cell Analysis (GCA) using Optical Coherence Tomography (OCT). GCA algorithm was used to measure the thickness of the overall avarage, minimum, superotemporal, superior, superonasal, inferonasal, inferior, inferotemporal GCIPL. Measurements of GCIPL thickness and central macular thickness of DM patients were compared with those of age and gender matched control group.
\end{abstract}

Results: The overall average, minimum, and the six sectors of GCIPL thickness measurements were significantly thinner in DM patients compared with healthy controls ( $p$ $<0.05$ ), but there was no significant difference between groups 1 and 2 ( $p>0.05$ ). In terms of duration of diabetes and DR status, there was no statistically significant difference in GCIPL parameters while correcting for age and presence of hypertension between the groups.

Conclusion: This study demonstrates a significant GCIPL thinning at all segments of the macula in DM patients than in healthy controls. In contrast, there was no significant difference between group 1 and 2 in terms of GCIPL thickness. These results confirm that DM has a neurodegenerative effect on the retina before vascular changes occur.

\section{Keywords}

Retinal ganglion cells, Diabetic retinopathy, Hypertension, Optical coherence tomography, Ganglion cell analysis

\section{Introduction}

Diabetic Retinopathy (DR) is a major cause of visual impairment in working age adults worldwide [1]. DR is clinically diagnosed with the onset of microvascular complications, such as microaneurysms, hemorrhages, and cotton-wool spots. Several studies have established that neurodegenerative changes occurred before the onset of microvascular complications in the earliest stages of diabetes by using electroretinography, perimetry, dark adaptation, contrast sensitivity, and color vision tests [2-7].

Optical Coherence Tomography (OCT) enables noninvasive, real-time visualizations of retinal morphologic features in many retinal diseases, including DR. Recent segmentation algorithms in spectral domain OCT (SDOCT) allow detailed screening of retinal layers. The Cirrus HD OCT (Carl Zeiss Meditec, Dublin, California, USA) can evaluate the Ganglion Cell-Inner Plexiform Layer (GCIPL) thickness of central macula using the Ganglion Cell Analysis (GCA).

The aim of this study is to ascertain whether Diabetes Mellitus (DM) affects the macular GCIPL thickness in patients who have Nonproliferative Diabetic Retinopathy (NPDR) or no DR using Cirrus HD-OCT and to consider the relationship between GCIPL measurements and age, sex, duration of diabetes, DR status, glycated Hemoglobin (HbA1c) level, Hypertension (HT) duration, and hypertensive retinopathy status. 


\section{Material and Methods}

This retrospective study was conducted to the provisions of the Declaration of Helsinki. Ethical approval was provided by the local Ethical Committee in December 2013. The medical registers of 108 eyes of $60 \mathrm{DM}$ patients and 104 eyes of 60 healthy subjects that were examined between July 2010 and November 2013 were analyzed. The patient group was divided into two subgroups: group 1 with no DR (56 eyes of 30 patients) and group 2 with NPDR ( 52 eyes of 30 patients). All eyes underwent a complete ophthalmic evaluation, including slit-lamp biomicroscopy and fundus examination.

Cases with an ocular history of trauma, surgery, laser procedure, and systemic disease except Type 2 DM and HT; significant media opacities; coexisting glaucomatous optic disc changes; neuro-ophthalmologic disorders; uveitis; retinal diseases that could affect the retinal nerve fibre layer; included macular degeneration, retinal vascular occlusion, macular edema, and spheric equivalent $>6$ diopters (D) were excluded. Age, gender, duration of diabetes and $\mathrm{HT}$, and mean serum $\mathrm{HbA} 1 \mathrm{C}$ level were gathered from the patient records. DM patients were seperated into three groups according to the duration of DM; $0-11,11-20$, and over 20 years.

The GCA algorithm was performed using macular cube $512 \times 128$ scan mode of Cirrus HD-OCT (Software version 6.0, Carl Zeiss Meditec, Inc.). Macular cube scans with signal strength better than 5 , and without eye movement artifacts were included the study. The macular GCIPL layer was measured automatically from outer boundary of the RNFL to the outer boundary of the Inner Plexiform Layer (IPL) using the GCA algorithm.

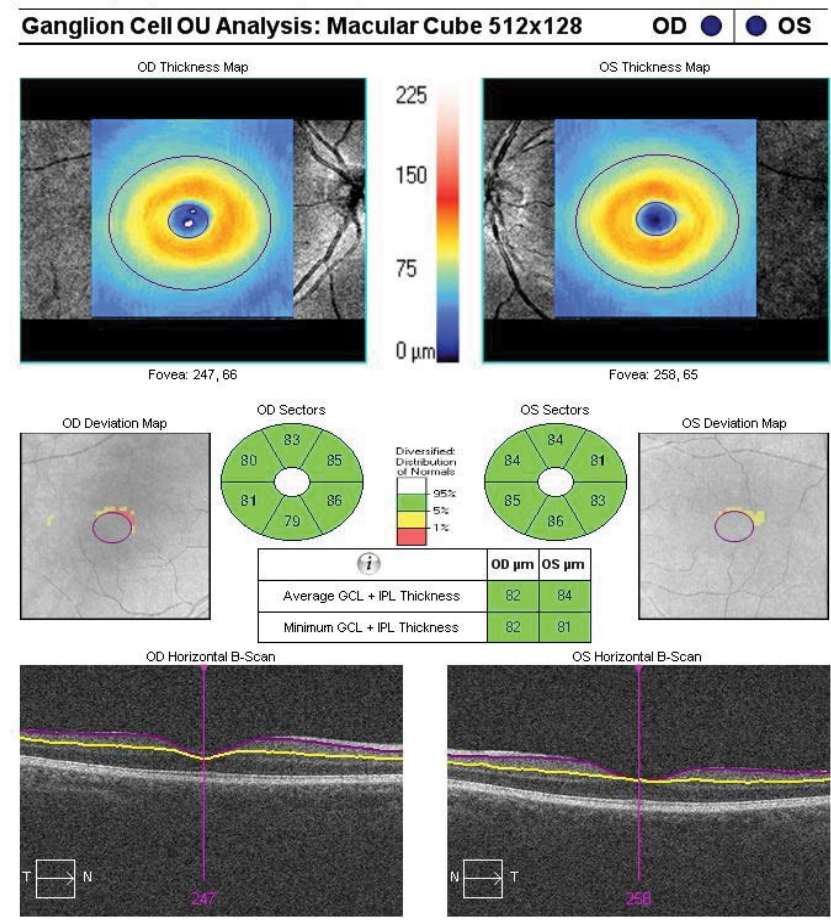

Figure 1: The Ganglion Cell Analysis (GCA) provided by the Cirrus OCT.
The average, minimum, and 6 sectoral (superotemporal, superior, superonasal, inferonasal, inferior, and inferotemporal) GCIPL values were measured in an elliptical ring around the fovea (Figure 1). The elliptical ring has a vertical inner and outer radius of 0.5 and $2.0 \mathrm{~mm}$, respectively, and a horizontal inner and outer radius of 0.6 and $2.4 \mathrm{~mm}$, respectively. The size of the inner ring was preferred to exclude the foveal area, where the Ganglion Cell Layer (GCL) is undetectable. The outer ring is horizontally extended by a factor of $20 \%$ to be matched the real anatomy of the macula.

Statistical analyses were implemented with the Statistical Package for the Social Sciences version 19.0 (SPSS, Inc., Chicago, IL, USA). The Kolmogorov-Smirnov $z$ test was performed to confirmed the normal distribution of variable groups, and the $\chi^{2}$ test was performed for the categorical variables. An independent sample $t$-test was used to compare the averages for parametric variables and the Mann-Whitney test was used for nonparametric variables. Pearson's and Spearman correlation analyses were performed, and a coefficient test was applied to determine the relationship between the findings. The differences between the groups were determined using Analysis of Covariance (ANCOVA) or all statistical tests, a $p<0.05$ and $p<0.01$ were considered significant. The Receiver-Operating Characteristic (ROC) curve analysis was performed to determine the cutoff value of $\mathrm{GCL}$ thickness.

Measurements of GCIPL and Central Macular Thickness (CMT) of DM patients and age- and sex-matched healthy controls were compared. Correlation analysis was used to find out the relationship between GCIPL thickness and age, sex, HbA1c level, DR status, duration of diabetes, HT duration, and hypertensive retinopathy status.

\section{Results}

Demographic, ocular, and systemic characteristics of $60 \mathrm{DM}$ patients and 60 healthy controls are shown in Table 1. Age of DM patients was between 40 and 84 years and that of healthy controls was between 40 and 85 years. There was no significant difference in age $(p$ $=0.181)$, sex $(p=0.100)$, Intraocular Pressure (IOP; $p$ $=0.925)$ and Best Corrected Visual Acuity (BCVA; $p=$ 0.181 ) between patients and healthy controls.

Glycemic control of patients was in average (mean $\mathrm{HbA} 1 \mathrm{c}, 8.22 \% \pm 1.76 \%$ ). Age was 40 to 80 years in group 1 and 41 to 84 years in group 2 . There was no significant difference in age $(p=0.134)$, sex $(p=0.118)$, IOP $(p=$ $0.780)$, and HbA1c level $(p=0.236)$ between groups 1 and 2. BCVA was significantly worse in group 2 than in group $1(p=0.045)$. Presence of HT was significantly different between DM patients and healthy controls $(p<$ 0.001 ), but there was no significant difference between groups 1 and 2 ( $p=0.405)$.

The average, minimum, and sectoral thicknesses 
Table 1: Demographic and clinical characteristics of the study population.

\begin{tabular}{|c|c|c|c|c|}
\hline Parameters & Controls & Patients with diabetes & Patients without DR & Patients with NPDR \\
\hline Number of cases $(n)$ & 60 & 60 & 30 & 30 \\
\hline Age (mean \pm SD) & $58.77 \pm 8.99$ & $62.01 \pm 11.26$ & $59.40 \pm 11.63$ & $64.63 \pm 10.41$ \\
\hline Gender (M:F) & $25: 35$ & $34: 26$ & $14: 16$ & $20: 10$ \\
\hline IOP, mmHg & $15.77 \pm 2.68$ & $15.65 \pm 2.61$ & $15.77 \pm 2.44$ & $15.52 \pm 2.81$ \\
\hline BCVA & $0.853 \pm 0.223$ & $0.896 \pm 0.191$ & $0.938 \pm 0.15$ & $0.852 \pm 0.22$ \\
\hline $\mathrm{HbA1c}(\%)$ & - & $8.22 \pm 1.76$ & $8.19 \pm 2.10$ & $8.24 \pm 1.38$ \\
\hline $\begin{array}{l}\text { Duration of diabetes (years) } \\
0-10 \\
11-20 \\
>20\end{array}$ & $\begin{array}{l}- \\
- \\
-\end{array}$ & $\begin{array}{l}37(61.7 \%) \\
15(25 \%) \\
8(13.3 \%)\end{array}$ & $\begin{array}{l}27(90 \%) \\
2(6.7 \%) \\
1(3.3 \%)\end{array}$ & $\begin{array}{l}10(33.3 \%) \\
13(43.3 \%) \\
7(23.3 \%)\end{array}$ \\
\hline Hypertension, n (\%) & $19(32 \%)$ & $41(68 \%)$ & $19(63 \%)$ & $22(73 \%)$ \\
\hline Duration of hypertension (years) & $11.36 \pm 10.47$ & $11.46 \pm 9.02$ & $10.21 \pm 9.59$ & $12.54 \pm 8.57$ \\
\hline $\begin{array}{l}\text { Grade of hypertensive retinopathy } \\
\text { Grade } 1 \\
\text { Grade } 2\end{array}$ & $\begin{array}{l}5(8.3 \%) \\
1(1.7 \%)\end{array}$ & $\begin{array}{l}14(23.3 \%) \\
8(13.3 \%)\end{array}$ & $\begin{array}{l}8(26.7 \%) \\
3(10 \%)\end{array}$ & $\begin{array}{l}6(20 \%) \\
5(16.7 \%)\end{array}$ \\
\hline
\end{tabular}

SD: Standart Deviation; M: Male; F: Female; IOP: Intraocular Pressure; BCVA: Best Corrected Visual Acuity; DR: Diabetic Retinopathy; NPDR: Nonproliferative Diabetic Retinopathy.

Table 2: Mean ganglion Cell-Inner plexiform layer thickness parameters in diabetic patients compared with healthy controls.

\begin{tabular}{|l|l|l|l|}
\hline Parameters & Patients with diabetes & Controls & p value \\
\hline Number of cases $(\mathrm{n})$ & 60 & 60 & \\
\hline Average GCIPL & $80.56 \pm 7.97$ & $83.70 \pm 5.93$ & 0.002 \\
\hline Minimum GCIPL & $74.56 \pm 12.25$ & $80.51 \pm 6.00$ & 0.000 \\
\hline Superotemporal GCIPL & $80.08 \pm 7.29$ & $82.71 \pm 5.67$ & 0.004 \\
\hline Superor GCIPL & $81.94 \pm 9.92$ & $84.71 \pm 6.51$ & 0.017 \\
\hline Superonasal GCIPL & $81.08 \pm 10.30$ & $85.01 \pm 7.06$ & 0.013 \\
\hline Inferonasal GCIPL & $79.99 \pm 9.36$ & $83.45 \pm 7.02$ & 0.009 \\
\hline Inferior GCIPL & $79.44 \pm 10.00$ & $82.58 \pm 6.97$ & 0.020 \\
\hline Inferotemporal GCIPL & $80.75 \pm 8.21$ & $83.81 \pm 5.80$ & 0.013 \\
\hline Central macular thickness & $253.15 \pm 23.15$ & $256.13 \pm 18.05$ & 0.053 \\
\hline
\end{tabular}

GCIPL: Ganglion Cell-Inner Plexiform Layer.

Table 3: Mean ganglion cell-inner plexiform layer thickness in diabetic patients without diabetic retinopthy compared with nonproliferative diabetic retinopathy.

\begin{tabular}{|l|l|l|l|}
\hline Parameters & Patients without DR & Patients with NDR & p value \\
\hline Number of cases & 30 & 30 & 0.387 \\
\hline Average GCIPL & $81.04 \pm 8.27$ & $80.04 \pm 7.69$ & 0.053 \\
\hline Minimum GCIPL & $77.21 \pm 9.84$ & $71.69 \pm 13.93$ & 0.882 \\
\hline Superotemporal GCIPL & $79.98 \pm 7.28$ & $80.19 \pm 7.38$ & 0.431 \\
\hline Superor GCIPL & $82.16 \pm 9.52$ & $81.69 \pm 10.43$ & 0.288 \\
\hline Superonasal GCIPL & $82.07 \pm 9.84$ & $80.02 \pm 10.78$ & 0.422 \\
\hline Inferonasal GCIPL & $80.75 \pm 9.15$ & $79.17 \pm 9.62$ & 0.311 \\
\hline Inferior GCIPL & $80.43 \pm 9.12$ & $78.38 \pm 10.86$ & 0.754 \\
\hline Inferotemporal GCIPL & $80.73 \pm 8.51$ & $80.77 \pm 7.95$ & 0.065 \\
\hline Central macular thickness & $250.50 \pm 25.66$ & $256.00 \pm 19.96$ & \\
\hline
\end{tabular}

of GCIPL (superotemporal, superior, superonasal, inferonasal, inferior, and inferotemporal) and CMT in all DM patients compared with those in healthy controls are shown in Table 2. All mean GCIPL thickness parameters were significantly thinner in DM patients than in healthy controls $(p<0.05)$. There was no significant difference in CMT between patients and healthy controls ( $p>0.05)$. There was no significant difference in GCIPL parameters and CMT between DM groups 1 and 2 ( $p>$ 0.05; Table 3).

Weak negative correlations were detected between age and average $(r=-0.428, p<0.001)$, minimum $(r$ $=-0.439, p<0.001)$, superotemporal $(r=-0.299, p=$
$0.002)$, superior $(r=-0.338, p<0.001)$, superonasal $(r$ $=-0.396, p<0.001)$, inferonasal $(r=-0.416, p<0.001)$, GCIPL inferior $(r=-0.424, p<0.001)$, and inferotemporal $(r=-0.335, p<0.001)$ GCIPL values in DM patients.

Weak negative correlations were detected between age and average $(r=-0.457, p<0.001)$, minimum $(r$ $=-0.466, p<0.001)$, superotemporal $(r=-0.257, p=$ $0.009)$, superior $(r=-0.404, p<0.001)$, superonasal $(r$ $=-0.477, p<0.001)$, inferonasal $(r=-0.466, p<0.001)$, inferior $(r=-0.324, p=0.001)$, and inferotemporal $(r=$ $0.385, p<0.001)$ GCIPL values in healthy controls. There was no significant correlation between age and CMT in DM patients $(r=0.132, p=0.172)$ and healthy controls 
$(r=-0.092, p=0.352)$. Figure 2 shows a weak negative correlation between GCIPL thickness and age in patients with diabetes and healthy controls.

There was no significant difference between sex and mean GCIPL parameters in DM patients $(p>0.05)$, but in healthy controls, superotemporal $(p=0.013)$ and inferotemporal ( $p=0.017) \mathrm{GCIPL}$ values were thicker in males. CMT was significantly thicker in males than in females in patients $(p=0.001)$ and healthy controls $(p=$ 0.02).

Weak positive correlations were observed between $\mathrm{HbA} 1 \mathrm{c}$ level and superotemporal GCIPL $(r=0.222, p=$ $0.021)$ and CMT $(r=0.209, p=0.03)$ in DM patients.

Presence of HT in patients with DM had a weak negative linear relationship with average $(r=-0.344, p<$ $0.0001)$, superior $(r=-0.264, p=0.006)$, superonasal $(r$ $=-0.274, p=0.004)$, inferonasal $(r=-0.304, p=0.001)$, and inferior $(r=-0.364, p<0.001) \mathrm{GCIPL}$. In healthy controls, there was no linear correlation between the presence of HT with any GCIPL parameter ( $p>0.05)$.

HT duration had a weak negative relationship with average $(r=-0.284, p=0.003)$, superotemporal $(r=-$ $0.198, p=0.040)$, inferonasal $(r=-0.244, p=0.011)$, inferior $(r=-0.346, p<0.001)$, and inferotemporal $(r$ $=-0.215, p=0.025)$ GCIPL in DM patients. Duration of

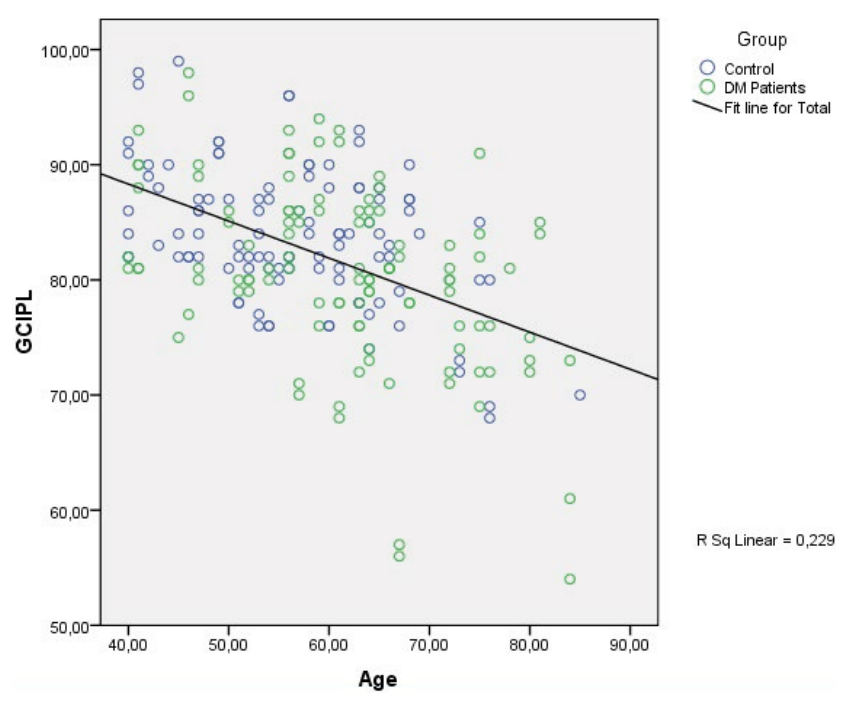

Figure 2: Correlation between GCIPL thickness and age in patients with DM and healthy controls $(p<0.001)$.
HT had a weak relationship with only GCIPL inferonasal $(r=-0.278, p=0.038)$ in healthy controls.

Stage of HT had a weak negative relationship with average $(r=-0.324, p=0.001)$, minimum $(r=-0.260, p$ $=0.007)$, superotemporal $(r=-0.267, p=0.005)$, superior $(r=-0.304, p=0.001)$, superonasal $(r=-0.312, p=$ $0.019)$, inferonasal $(r=-0.288, p=0.002)$, and inferior $(r=-0.318, p=0.001) \mathrm{GCIPL}$ in DM patients. There was no linear relationship between the stage of $\mathrm{HT}$ with any GCIPL parameter $(p>0.05)$ in healthy controls. There was no linear relationship between CMT with presence, duration, or stage of HT ( $p>0.05)$.

To correct the effects of age and the presence of HT, ANCOVA was performed to assess the differences between groups. Taking age and presence of HT as covariates, average $(p=0.01)$, minimum $(p<0.001)$, superotemporal $(p=0.044)$, superonasal $(p=0.003)$, inferonasal $(p=0.007)$, and inferotemporal $(p=0.019)$ GCIPL parameters still were significantly thinner in DM patients than in healthy controls, but there were no significant differences in superior $(p=0.115)$ and inferior ( $p=0.065)$ GCIPL.

In terms of duration of diabetes, there was no statistically significant difference in GCIPL parameters while correcting for age and presence of HT between the groups (Table 4). There was no statistically significant difference in GCIPL parameters while correcting for age and presence of HT between patients with no DR and those with NPDR (Table 5).

The Area Under the ROC Curve (AUC) for average GCIPL was calculated as $0.624(p<0.05)$. This value shows that the average GCIPL parameter was correctly classified as $62.4 \%$ in patients with and without diabetes. In the analysis, the sensitivity and the specificity values were examined, and an optimum point was selected as a cutoff value. The sensitivity and specificity for a cutoff point of $81.5 \mu \mathrm{m}$ were $58 \%$ and $68 \%$, respectively (Figure 3). Cases with an average GCIPL value under $81.5 \mu \mathrm{m}$ are more likely to have diabetes.

\section{Discussion}

DR is a common complication of diabetes. Although many studies have focused on vascular complications

Table 4: Mean corrected ganglion cell-inner plexiform layer thickness parameters between the groups in terms of duration of diabetes.

\begin{tabular}{|c|c|c|c|c|c|}
\hline Parameters & $0-10$ years & $11-20$ years & $>20$ years & $\mathbf{F}$ & $p$ value \\
\hline Average GCIPL & $80.19 \pm 0.89$ & $80.53 \pm 1.50$ & $82.73 \pm 2.13$ & 0.589 & 0.557 \\
\hline Minimum GCIPL & $76.24 \pm 1.36$ & $69.98 \pm 2.28$ & $74.61 \pm 3.24$ & 2.576 & 0.081 \\
\hline Superotemporal GCIPL & $79.58 \pm 0.86$ & $79.65 \pm 1.44$ & $84.18 \pm 2.05$ & 2.227 & 0.113 \\
\hline Superor GCIPL & $81.08 \pm 1.16$ & $82.31 \pm 1.95$ & $86.09 \pm 2.77$ & 1.341 & 0.266 \\
\hline Superonasal GCIPL & $81.02 \pm 1.15$ & $79.03 \pm 1.94$ & $85.86 \pm 2.75$ & 2.145 & 0.122 \\
\hline Inferonasal GCIPL & $79.44 \pm 1.05$ & $80.02 \pm 1.76$ & $82.02 \pm 2.50$ & 0.472 & 0.625 \\
\hline Inferior GCIPL & $79.35 \pm 1.15$ & $80.02 \pm 1.93$ & $78.70 \pm 2.74$ & 0.087 & 0.917 \\
\hline Inferotemporal GCIPL & $80.36 \pm 0.96$ & $82.05 \pm 1.61$ & $80.17 \pm 2.28$ & 0.431 & 0.651 \\
\hline
\end{tabular}

GCIPL: Ganglion Cell-Inner Plexiform Layer. 
of diabetes, it is becoming obvious that neurodegenerative problems occur before manifest signs of vascular lesions $[7,8]$.

A study of streptozotocin-induced diabetic rats showed that the retinal ganglion cell bodies were decreased by $10 \%$ after 8 months of diabetes [9]. An immunohistochemistry study with streptozotocin-diabetic rats showed $20 \%$ diminution in the NeuN-positive retinal ganglion cells after 4 months of diabetes [10]. A previous study showed that hyperglycemia increases O-GlcNAcylation in GCL and Inner Nuclear Layer (INL) in STZ-induced diabetic mice retinas and increased O-GIcNAcylation is associated with RGC death in diabetic retinas [11]. These studies revealed that RGC apoptosis begins soon after the onset of diabetes. Hyperglycemia, oxidative stress and advanced glycation end products are some of the causes of RGC apoptosis.

Van Dijk, et al. [12] also showed that GCIPL and INL of DM patients with minimal DR were significantly thinner in the pericentral and peripheral areas than in healthy

\section{ROC Curve}

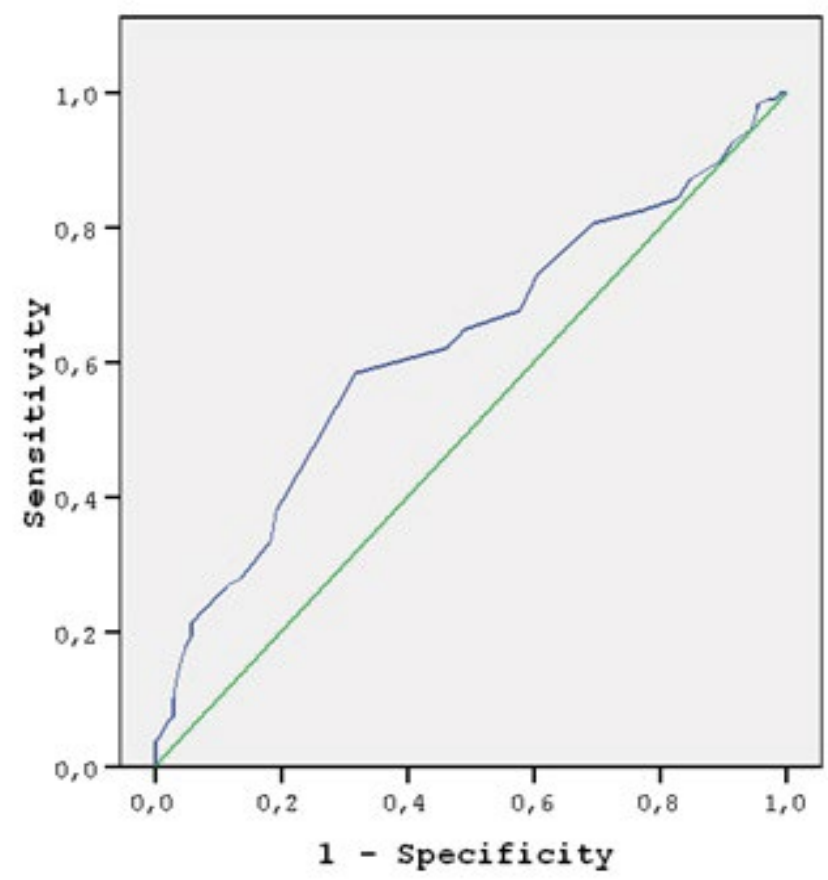

Figure 3: ROC Curve of average ganglion cell-inner plexiform layer thickness. controls. There were no significant differences in any layer thickness between DM patients without DR and healthy controls. The quality of OCT images has considerably improved with the advent of SD-OCTs, allowing better segmentation and delineation of various macular layers. A previous study with SD-OCT similarly demonstrated that RGC nucleus and dendritis are affected in DM patients' eyes with no apparent sign of diabetic vascular changes [13].

In our study, all GCIPL thickness parameters were thinner in DM patients compared with healthy controls, but there was no significant difference between DM patients without DR and with NPDR. The results support early neurodegenerative effects of diabetes; the GCIPL changes occur before the vascular clinical signs.

The threshold between healthy and affected Retinal Thickness (RT) is not clear because of the high interindividual variability in RT. Thus, RT measurements using Stratus OCT were not capable for detecting early neurodegeneration [12]. In the current study, AUC was calculated as 0.624 for average GCIPL $(p<0.05)$. This value shows that the average GCIPL parameter correctly classifies a $62.4 \%$ rate in patients with and without diabetes. Cases with average GCIPL value under $81.5 \mu \mathrm{m}$ are more likely to have diabetes.

Previous studies showed a linear relationship between decreased GCL thickness with older age $[14,15]$. Our results showed weak negative correlations between age and all GCIPL parameters. The results from previous studies about the relationship between GCIPL thickness and sex vary. Mwanza, et al. [16] revealed that thinner GCIPL thickness is significantly related with older age and male gender. Koh, et al. [14] concluded that thinner GCIPL was related with older age and female gender independently. In our study, average GCIPL values were six sectors thicker in females but it was not statistically significant.

Diabetes and HT frequently occur together. There is substantial overlap between diabetes and HT in etiology and disease mechanisms [17]. Publications exploring the relationship between DR and HT vary. In the Wisconsin Epidemiologic Study of Diabetic Retinopathy (WESDR), diastolic blood pressure was an important risk factor of progression of DR in type 1 DM patients [18].

Table 5: Mean corrected ganglion cell-inner plexiform layer thickness parameters in diabetic patients without diabetic retinopathy compared with nonproliferative diabetic retinopathy

\begin{tabular}{|c|c|c|c|c|}
\hline Parameters & Patients without DR & Patients with NPDR & $\mathbf{F}$ & p value \\
\hline Average GCIPL & $80.37 \pm 0.97$ & $80.75 \pm 1.01$ & 0.072 & 0.789 \\
\hline Minimum GCIPL & $76.34 \pm 1.48$ & $72.62 \pm 1.54$ & 2.947 & 0.089 \\
\hline Superotemporal GCIPL & $79.56 \pm 0.94$ & $80.63 \pm 0.98$ & 0.599 & 0.441 \\
\hline Superor GCIPL & $81.54 \pm 1.27$ & $82.35 \pm 1.32$ & 0.193 & 0.661 \\
\hline Superonasal GCIPL & $81.28 \pm 1.27$ & $80.86 \pm 1.32$ & 0.049 & 0.825 \\
\hline Inferonasal GCIPL & $79.96 \pm 1.14$ & $80.01 \pm 1.18$ & 0.001 & 0.976 \\
\hline Inferior GCIPL & $79.69 \pm 1.24$ & $79.17 \pm 1.29$ & 0.083 & 0.774 \\
\hline Inferotemporal GCIPL & $80.15 \pm 1.03$ & $81.39 \pm 1.07$ & 0.681 & 0.411 \\
\hline
\end{tabular}

GCIPL: Ganglion Cell-Inner Plexiform Layer; DR: Diabetic Retinopthy; NPDR: Nonproliferative Diabetic Retinopathy. 
However, older onset type 2 DM patients, systolic and diastolic blood pressure and HT status were not related with the DR progression [19]. Tham, et al. [20] concluded that narrower arteriolar and venular diameter, and decreased vascular tortuosity were related with thinner GCIPL thickness. Vascular tortuosity is associated with the properties of the endothelium. The endothelium increases vascular tortuosity by secreting nitric oxide and endothelin and, autoregulates tissue perfusion [21-23]. However, decreased vascular tortuosity leads to retinal ganglion cell degeneration due to poor tissue nourishment [20]. In our study, presence of HT in the DM patients had a weak negative linear correlation with average, superior, superonasal, inferonasal, and inferior GCIPL thicknesses. In healthy controls, there was no linear correlation between the presence of HT with any GCIPL parameter. Duration of HT had a weak negative relationship with average, superotemporal, inferonasal, inferior, and inferotemporal GCIPL thicknesses in DM patients. Duration of HT had a weak relationship with only inferonasal GCIPL in healthy controls. Stage of HT had a weak negative relationship with average, minimum, superotemporal, superior, superonasal, inferonasal, and inferior GCIPL thickness in DM patients. There was no relationship between the stage of HT and GCIPL parameters in healthy controls.

The most important predisposing factor for the development and progression of DR is the duration of diabetes. In the WESDR, the prevalence of DR varied from $28.8 \%$ to $77.8 \%$ in DM patients for $<5$ and $\geq 15$ years, respectively [24]. Van Dijk, et al. [25] showed a significant inverse linear correlation between $\mathrm{GCL}$ thickness and duration of diabetes.

In the current study, in terms of duration of diabetes and DR status, there was no statistically significant difference in GCIPL parameters while correcting for age and presence of HT between the groups. We acknowledge that our study has a shortcoming that the absence of a longitudinal follow-up to assess possible long-term changes in GCL.

Finally, our results showed that ganglion cells have been affected and GCL thickness decreases in patients with DR, even in the very early stages. Presence and stage of HT can act on ganglion cells, particularly in patients with diabetes and HT together. Therefore, we corrected for the presence of the HT effect, and GCIPL parameters were still observed to be significantly thinner in DM patients than in healthy controls. It can be concluded that ganglion cells may decrease more in patients with diabetes and HT together or in advanced DR. Consequently, in patients with DR, analysis of the ganglion cells helps us to find out the etiology of patients who have cured anatomically but still have restricted visual improvement.

\section{References}

1. Klein BE (2007) Overview of epidemiologic studies of dia- betic retinopathy. Ophthalmic Epidemiol 14: 179-183.

2. Dosso AA, Bonvin ER, Morel Y, Golay A, Assal JP, et al. (1996) Risk factors associated with contrast sensitivity loss in diabetic patients. Graefes Arch Clin Exp Ophthalmol 234: 300-305.

3. Hardy KJ, Lipton J, Scase MO, Foster DH, Scarpello JH (1992) Detection of colour vision abnormalities in uncomplicated type 1 diabetic patients with angiographically normal retinas. $\mathrm{Br} \mathrm{J}$ Ophthalmol 76: 461-464.

4. Lopes de Faria JM, Katsumi O, Cagliero E, Nathan D, Hirose $T$ (2001) Neurovisual abnormalities preceding the retinopathy in patients with long-term type 1 diabetes mellitus. Graefes Arch Clin Exp Ophthalmol 239: 643-648.

5. Ng JS, Bearse MA Jr, Schneck ME, Barez S, Adams AJ (2008) Local diabetic retinopathy prediction by multifocal ERG delays over 3 years. Invest Ophthalmol Vis Sci 49: 1622-1628.

6. van Dijk HW, Verbraak FD, Stehouwer M, Kok PH, Garvin MK, et al. (2011) Association of visual function and ganglion cell layer thickness in patients with diabetes mellitus type 1 and no or minimal diabetic retinopathy. Vision Res 51: 224-228.

7. Antonetti DA, Klein R, Gardner TW (2012) Diabetic retinopathy. N Engl J Med 366: 1227-1239.

8. Cunha-Vaz JG (2015) Diabetic Retinopathy: Need for More Research to Understand the Relative Role of Neuropathy and Microvascular Disease. Ophthalmic Res 54: 109-111.

9. Barber AJ, Lieth E, Khin SA, Antonetti DA, Buchanan AG, et al. (1998) Neural apoptosis in the retina during experimental and human diabetes. Early onset and effect of insulin. J Clin Invest 102: 783-791.

10. Zeng XX, Ng YK, Ling EA (2000) Neuronal and microglial response in the retina of streptozotocin-induced diabetic rats. Vis Neurosci 17: 463-471.

11. Kim SJ, Yoo WS, Choi M, Chung I, Yoo JM, et al. (2016) Increased O-GIcNAcylation of NF-kB enhances retinal ganglion cell death in streptozotocin-induced diabetic retinopathy. Curr Eye Res 19: 1-9.

12. van Dijk HW, Kok PH, Garvin M, Sonka M, Devries JH, et al. (2009) Selective loss of inner retinal layer thickness in type 1 diabetic patients with minimal diabetic retinopathy. Invest Ophthalmol Vis Sci 50: 3404-3409.

13. Chhablani J, Sharma A, Goud A, Peguda HK, Rao HL, et al. (2015) Neurodegeneration in type 2 diabetes: evidence from spectral-domain optical coherence tomography. Invest Ophthalmol Vis Sci 56: 6333-6338.

14. Koh VT, Tham YC, Cheung CY, Wong WL, Baskaran M, et al. (2012) Determinants of ganglion cell-inner plexiform layer thickness measured by high-definition optical coherence tomography. Invest Ophthalmol Vis Sci 53: 5853-5859.

15. Gao H, Hollyfield JG (1992) Aging of the human retina. Differential loss of neurons and retinal pigment epithelial cells. Invest Ophthalmol Vis Sci 33: 1-17.

16. Mwanza JC, Durbin MK, Budenz DL, Girkin CA, Leung CK, et al. (2011) Cirrus OCT Normative Database Study Group. Profile and predictors of normal ganglion cell-inner plexiform layer thickness measured with frequency-domain optical coherence tomography. Invest Ophthalmol Vis Sci 52: 7872-7879.

17. Cheung BM, Li C (2012) Diabetes and hypertension: is there a common metabolic pathway? Curr Atheroscler Rep 14: $160-166$. 
18. Klein R, Klein BE, Moss SE, Cruickshanks KJ (1998) The Wisconsin Epidemiologic Study of Diabetic Retinopathy: XVII. The 14 year incidence and progression of diabetic retinopathy and associated risk factors in type 1 diabetes. Ophthalmology 105: 1801-1815.

19. Klein R, Klein BE, Moss SE, Cruickshanks KJ (1994) Relationship of hyperglycemia to the long-term incidence and progression of diabetic retinopathy. Arch Intern Med 154: 2169-2178.

20. Tham YC, Cheng CY, Zheng Y, Aung T, Wong TY, et al. (2013) Relationship between retinal vascular geometry with retinal nerve fiber layer and ganglion cell-inner plexiform layer in nonglaucomatous eyes. Invest Ophthalmol Vis Sci 54: 7309-7316.

21. Gidday JM, Zhu Y (1998) Endothelium-dependent changes in retinal blood flow following ischemia. Curr Eye Res 17: 798-807.
22. Tomita $Y$, Kubis $N$, Calando $Y$, Tran Dinh $A$, Méric $P$, et al. (2005) Long-term in vivo investigation of mouse cerebral microcirculation by fluorescence confocal microscopy in the area of focal ischemia. J Cereb Blood Flow Metab 25: 858-867.

23. Goldman D, Popel AS (2000) A computational study of the effect of capillary network anastomoses and tortuosity on oxygen transport. J Theor Biol 206: 181-194.

24. Klein R, Klein BE, Moss SE, Davis MD, DeMets DL (1984) The Wisconsin epidemiologic study of diabetic retinopathy. III. Prevalence and risk of diabetic retinopathy when age at diagnosis is 30 or more years. Arch Ophthalmol 102: 527532.

25. van Dijk HW, Verbraak FD, Kok PH, Garvin MK, Sonka M, et al. (2010) Decreased retinal ganglion cell layer thickness in patients with type 1 diabetes. Invest Ophthalmol Vis Sci 51: 3660-3665. 